\title{
Einfluss der Aufschlusstemperatur auf die morphologischen Eigenschaften von TMP aus Kiefernholz
}

\author{
E. Roffael · G. Bär $\cdot$ C. Behn $\cdot$ B. Dix
}

Published online: 3 Oktober 2008

(C) Die Autoren 2008. Dieser Artikel wurde mit Open Access auf Springerlink.com veröffentlicht.

\section{Effect of pulping temperature on the morphological properties of TMP made from pine wood}

Subject Chips from pine wood were subjected to thermomechanical pulping (TMP) at 140 and $180^{\circ} \mathrm{C}$ for 5 minutes, whereas the cooked chips were defibrated using a single disk pressurized refiner at the same temperatures (140 and $\left.180^{\circ} \mathrm{C}\right)$. The fibres were tested for some of their morphological properties including fibre length, fibre width, cellwall thickness. Moreover, the fine fibre fraction (zero fibres) and the content of splinters were also estimated. The results reveal, that increasing the temperature during thermomechanical pulping decreases the fibre length, the cell width

E. Roffael (『)

Georg-August-Universität Göttingen,

Büsgenweg 4,

37077 Göttingen, Deutschland

e-mail: eroffae1@gwdg.de

G. Bär

Institut für Zellstoff und Papier (PTS-IZP),

Papiertechnische Stiftung (PTS),

Pirnaer Str. 37,

01809 Heidenau, Deutschland

C. Behn

Büsgen-Institut, Abteilung Molekulare Holzbiotechnologie und Technische Mykologie,

Georg-August-Universität Göttingen,

Büsgenweg 2,

37077 Göttingen, Deutschland

B. Dix

Wilhelm-Klauditz-Institut (WKI),

Fraunhofer-Institut für Holzforschung,

Bienroder Weg $54 \mathrm{E}$,

38108 Braunschweig, Deutschland

e-mail: brigitte.dix@wki.fraunhofer.de and the fibre wall thickness. It also increases the amount of fine fibres and increases the curl factor.

\section{Material und Methodik}

Kiefernholzhackschnitzel wurden nach dem TMP-Verfahren bei 140 und $180^{\circ} \mathrm{C}$ für 5 Minuten thermisch aufgeschlossen. Die Zerfaserung erfolgte in einem Einscheibenrefiner mit einem Scheibenabstand von $0,3 \mathrm{~mm}$ bei jeweils 140 und $180^{\circ} \mathrm{C}$. Die so erzeugten Fasern wurden mit Hilfe des Faserstoffanalysegerätes (Mod. FibreLab, Fa. Metso) auf einige ihrer morphologischen Eigenschaften hin untersucht. Die Ergebnisse sind in Tabelle 1 zusammengestellt.

\section{Ergebnisse und Diskussion}

Es wird deutlich, dass eine Erhöhung der Aufschlusstemperatur von 140 auf $180^{\circ} \mathrm{C}$ die Faserlänge von 1,88 auf $1,68 \mathrm{~mm}$ verkürzt und den Faserdurchmesser von 34,7 auf $30,9 \mu \mathrm{m}$ verringerte. Ebenfalls nahm die Faserwandstärke hierdurch von 12,4 auf 11,5 $\mu \mathrm{m}$ ab. Der Feinanteil erhöhte sich von 14,79 auf 22,92\%. Die Zunahme des Feinanteils steht möglicherweise mit der oft in der Fachliteratur getroffenen Feststellung in Zusammenhang, dass durch eine exzessive Erhöhung der Aufschlusstemperatur eine Abnahme der Biegefestigkeit der hergestellten MDF einhergeht (vgl. z.B. Schneider 1999).

Danksagung Das Verbundvorhaben zwischen der Universität Göttingen, dem Fraunhofer-Institut für Holzforschung und dem Institut für Zellstoff und Papier der Papiertechnischen Stiftung wurde mit Mitteln des Bundesministeriums für Wirtschaft und Technologie (BMWi) durch die Arbeitsgemeinschaft industrieller Forschungsver- 
Tabelle 1 Morphologische Eigenschaften von Faserstoffen aus Kiefernholz in Abhängigkeit von der Aufschlusstemperatur ( 140 und $180^{\circ} \mathrm{C}$ )

Table 1 Morphological properties of TMP from pine wood prepared at pulping temperatures of 140 and $180{ }^{\circ} \mathrm{C}$

\begin{tabular}{|c|c|c|c|c|c|c|}
\hline Faserstoff & $\begin{array}{c}\text { Splitter- } \\
\text { anteil } \\
\%\end{array}$ & $\begin{array}{c}\text { Faserlänge } \\
\text { mm }\end{array}$ & $\begin{array}{c}\text { Faser- } \\
\text { durchmesser } \\
\mu \mathrm{m}\end{array}$ & $\begin{array}{c}\text { Faserwand- } \\
\text { stärke } \\
\mu \mathrm{m}\end{array}$ & $\begin{array}{c}\text { Feinanteil } \\
\%\end{array}$ & $\begin{array}{c}\text { Kräusel- } \\
\text { faktor } \\
\%\end{array}$ \\
\hline $\mathrm{TMP} 140^{\circ} \mathrm{C}, 5 \mathrm{~min}$ & 41,8 & $\begin{array}{l}1,87 \\
1,89 \\
\mathbf{1 , 8 8}\end{array}$ & $\begin{array}{l}34,9 \\
34,4 \\
\mathbf{3 4 , 7}\end{array}$ & $\begin{array}{l}12,5 \\
12,2 \\
\mathbf{1 2 , 4}\end{array}$ & $\begin{array}{l}13,98 \\
15,60 \\
\mathbf{1 4 , 7 9}\end{array}$ & $\begin{array}{l}8,0 \\
7,5 \\
7,8\end{array}$ \\
\hline $\mathrm{TMP} 180^{\circ} \mathrm{C}, 5 \mathrm{~min}$ & 40,1 & $\begin{array}{l}1,67 \\
1,68 \\
\mathbf{1 , 6 8}\end{array}$ & $\begin{array}{l}30,9 \\
30,8 \\
\mathbf{3 0 , 9}\end{array}$ & $\begin{array}{l}11,5 \\
11,5 \\
\mathbf{1 1 , 5}\end{array}$ & $\begin{array}{l}22,29 \\
23,54 \\
\mathbf{2 2 , 9 2}\end{array}$ & $\begin{array}{l}12,1 \\
11,9 \\
\mathbf{1 2 , 0}\end{array}$ \\
\hline
\end{tabular}

einigungen (AiF- Nr. 14801 BG) über den Internationalen Verein für Technische Holzfragen (iVTH) gefördert.

Open Access Dieser Artikel wird zu den Bedingungen der "Creative Commons Attribution Noncommercial License" zur Verfügung gestellt. Damit ist eine nichtkommerzielle Nutzung, Verbreitung und Vervielfältigung erlaubt, sofern die Autoren des Artikels und die genaue Quelle angegeben sind.

\section{Literatur}

Schneider T (1999) Untersuchungen über den Einfluss von Aufschlussbedingungen des Holzes und der Faserstofftrocknung auf die Eigenschaften von mitteldichten Faserplatten. Dissertation an der Fakultät für Forstwissenschaften und Waldökologie der Georg-August-Universität Göttingen, Shaker Verlag, Aachen, S. 84 